\title{
Nanohertz gravitational waves from NEC violation in the early universe
}

\author{
Hiroaki W. H. Tahara ${ }^{1, *}$ and Tsutomu Kobayashi ${ }^{1, \dagger}$ \\ ${ }^{1}$ Department of Physics, Rikkyo University, Toshima, Tokyo 171-8501, Japan
}

\begin{abstract}
We study nanohertz gravitational waves relevant to pulsar timing array experiments from quantum fluctuations in the early universe with null energy condition (NEC) violation. The NEC violation admits accelerated expansion with the scale factor $a \propto(-t)^{-p}(p>0)$, which gives the tensor spectral index $n_{t}=2 /(p+1)>0$. To evade the constraint from Big Bang nucleosynthesis (BBN), we connect the NEC-violating phase to a subsequent short slow-roll inflationary phase which ends with standard reheating, and thereby reduce the high frequency part of the spectrum. An explicit model is constructed within the cubic Horndeski theory which allows for stable violation of the NEC. We present numerical examples of the background evolution having the different maximal Hubble parameters (which determine the peak amplitude of gravitational waves), the different inflationary Hubble parameters (which determine the amplitudes of high frequency gravitational waves), and different durations of the inflationary phase (which essentially determine the peak frequency of the spectrum). We display the spectra with $n_{t}=0.8,0.9$, and 0.95 for $f \lesssim 1 / \mathrm{yr}$, which are consistent with the recent NANOGrav result. We also check that they do not contradict the BBN constraint. We discuss how the nearly scale-invariant spectrum of curvature perturbations is produced in the NEC-violating phase.
\end{abstract}

\section{INTRODUCTION}

The stochastic gravitational wave background is of great interest of cosmologists and astrophysicists since the detection of its power spectrum reveals to us highenergy events and their unknown physics in the early universe. The power spectrum for $f \lesssim 10^{-15} \mathrm{~Hz}$ is constrained by experiments of the cosmic microwave background $(\mathrm{CMB})$, whereas that in the high-frequency range is constrained by experiments of pulsar-timing arrays and space/ground-based interferometers. Recently, the NANOGrav collaboration reported that they found a strong preference for a stochastic common-spectrum process in their 12.5-yr data set [1] although definitive evidence of quadrupolar interpulsar correlations [2] is lacked. If the common-spectrum process is due to supermassive black hole binaries, it would be the first evidence of their formation and coalescence. Other possible explanations for the NANOGrav result have been proposed by recent studies [3-21] from the view point of cosmic strings [3-6], dark phase transition [7-9], QCD phase transition $[10,11]$, and second-order gravitational waves from primordial black holes [12-16] or resonant amplification of other fields [17, 18].

Among a variety of possibilities, in this paper we discuss whether it is possible to generate gravitational waves of inflationary origin [22] that have amplitudes large enough to be detected in the nanohertz range with pulsar timing arrays while evading other observational constraints. Taking the tensor amplitude and spectral index simply as parameters, one can derive the region of the parameter space consistent with the NANOGrav result at $f \sim 1 /$ yr and the Planck limit at $f \lesssim 10^{-15} \mathrm{~Hz}$. However, if one assumes that the gravitational wave energy

\footnotetext{
* Email: tahara" at"rikkyo.ac.jp

† Email: tsutomu" at" rikkyo.ac.jp
}

density $\Omega_{\mathrm{GW}}(f)$ has a single power-law form with $n_{t} \sim 1$ up to the high frequency end of the spectrum, Big Bang nucleosynthesis (BBN) then excludes the entire parameter space [20]. To explore the inflationary explanation for the NANOGrav result, we therefore have to resolve the following issues. First of all, it is difficult to produce such an extremely blue gravitational wave spectrum directly from inflation. A blue inflationary gravitational wave spectrum implies the violation of the null energy condition (NEC), which usually results in some kinds of instabilities. Even if stable violation of the NEC were possible, generating an extremely blue spectrum with $n_{t} \sim 1$ would be even more difficult because the Hubble expansion rate that determines the tensor amplitude is supposed to be nearly constant during inflation. Furthermore, the spectrum must be less steep for $f \gtrsim 1 / \mathrm{yr}$ to evade the BBN constraint [23].

At the level of the background solution, one can obtain NEC-violating inflation (super-inflation, $\dot{H}>0$ ) with a phantom scalar field [24-28] or a k-essence field [29]. Unfortunately, at the level of linear perturbations all of these earlier models are unstable. It was, however, pointed out that stable violation of the NEC is possible in a consistent effective field theory [30]. Indeed, galileon and Horndeski theories [31-34] admit stable NEC-violating inflation [35, 36]. (See also [37] for a higher-derivative scalar-field theory admitting a similar super-accelerating phase.) In the same context, a more radical scenario has been proposed in which the universe starts expanding from Minkowski (galilean genesis) [38]. Variants of the galilean genesis scenario are found in [39-52]. Some of these introduce an NEC-violating phase connected smoothly to a subsequent quasi-de Sitter inflationary phase [46, 48]. See [53] for a review on NEC violation and its cosmological consequences. An NEC-violating inflationary solution has also been considered in different contexts such as loop quantum gravity $[54,55]$.

In the above NEC-violating examples of the early 
universe scenarios, gravitational waves originated from quantum fluctuations of the metric typically have a blue spectrum. This is because the amplitude of the metric perturbations is basically given by $h_{i j} \sim H / M_{\mathrm{Pl}}$, where $H$ is the Hubble parameter at the horizon crossing time (which is increasing when the NEC is violated) and $M_{\mathrm{Pl}}$ is the Planck mass. Note, however, that its increasing rate, $\dot{H} / H^{2}$, is small in quasi-de Sitter inflation and consequently the tensor spectral index $n_{t}$ is much smaller than one. (See, e.g., $[56,57]$ for recent analyses of a blue tensor tilt from slow-roll inflation.) Therefore, the expansion of the early universe must be accelerating and significantly away from de Sitter to produce gravitational waves with $n_{t} \sim 1$. (There is a possibility that the primordial tensor spectrum is blue-tilted due to the time variation of the tensor propagation speed [58, 59], but its effect can be absorbed entirely into the time variation of the Hubble parameter in the Einstein frame [60]. A non-standard symmetry-breaking pattern [61-66] and a non-Bunch-Davis initial state [67] can also yield a blue tensor tilt.)

The purpose of the present paper is to propose an early universe scenario that can generate large primordial gravitational waves in the nanohertz range and at the same time is consistent with other observations. Our scenario is composed of NEC-violating super-inflation and a subsequent (relatively short) phase of NEC-preserving slowroll inflation. We construct an explicit model within the cubic Horndeski theory. Our scenario is closely related to, but different in many aspects from, those in $[46,48]$.

This paper is organized as follows. In the next section, we give a brief sketch of the scenario. We then present in more detail the explicit Lagrangian for our model and study the background cosmological evolution in Sec. III. The gravitational wave spectrum generated in our model is presented in Sec. IV. The mechanism for producing scalar perturbations is discussed in Sec. V. Section VI is devoted to conclusions.

\section{SKETCH OF THE IDEA}

Let us begin with introducing our basic idea for generating an extremely blue tensor spectrum in the early universe. Our scenario is composed of the following three phases: an NEC-violating phase, subsequent relatively short inflation, and (standard) reheating. Each phase is characterized by the different dynamics of a scalar field in the potential, as shown schematically in Fig. 1.

The scale factor and the Hubble parameter during the NEC-violating phase we consider are given, respectively, by

$$
a \propto(-t)^{-p}, \quad H=\frac{p}{-t},
$$

where $t(<0)$ is the proper time and $p(>0)$ is a constant. Clearly, the NEC is violated: $\dot{H}=p /(-t)^{2}>0$. Note that in this phase the universe undergoes an accelerated

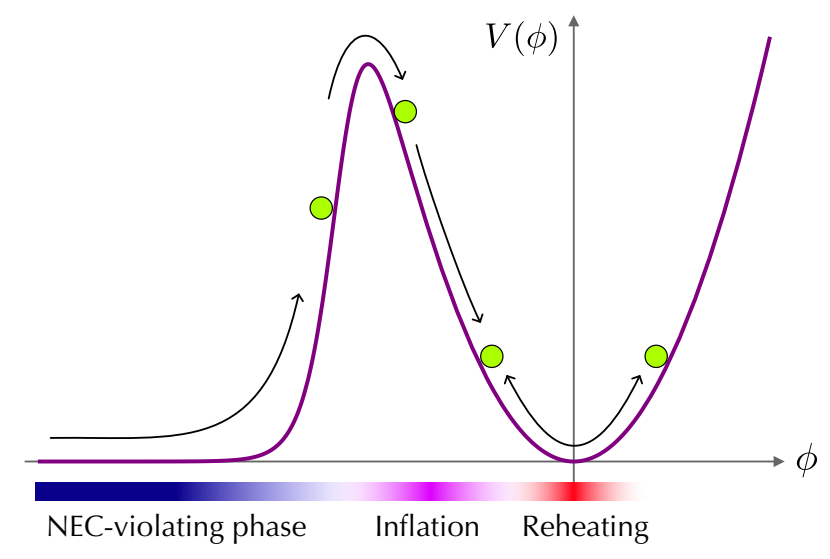

FIG. 1. The shape of the potential and the dynamics of the scalar field in our scenario.

expansion, $\ddot{a} / a=p(1+p) /(-t)^{2}>0$, so that the horizon and flatness problems are resolved in the same way as in standard quasi-de Sitter inflation. The amplitude of tensor modes is basically determined by the Hubble parameter at horizon crossing. In this NEC-violating phase where $H$ is increasing with time as in Eq. (1), the tensor spectral index is given by

$$
n_{t}=\frac{2}{p+1}
$$

and hence $0<n_{t}<2$ for $p>0$.

The cosmic expansion with $\dot{H}>0$ is caused by a scalar field having (effectively) negative kinetic energy and climbing up the potential. It is likely that the curvature perturbation exhibits ghost/gradient instabilities around such a background, which indeed occurs in $P\left(\phi,(\partial \phi)^{2}\right)$ theory. However, by adding a galileon-type interaction term $\sim(\partial \phi)^{2} \square \phi$ to the Lagrangian, one can stabilize such an NEC-violating background, as is demonstrated in Refs. [35, 36, 38]. We also use this idea to stabilize the NEC-violating phase.

The first phase described above ends when the scalar field goes over the top of the potential. The Hubble parameter takes the maximum value $H_{*}$ at that moment. The scalar field then rolls down the potential and quasi-de Sitter inflation occurs. This inflationary phase is rather standard except that its duration is relatively short. The tensor modes generated during this phase have a nearly flat spectrum. Finally, the scalar field starts to oscillate around the bottom of the potential and thereby the reheating process proceeds in a standard way.

In the next section we present a concrete model realizing the entire cosmological evolution of this scenario. 


\section{MODEL}

In this section, we seek for the Lagrangian that admits the aforementioned early universe scenario within the following subclass of the Horndeski theory:

$$
\mathcal{L}=G_{2}(\phi, X)-G_{3}(\phi, X) \square \phi+\frac{M_{\mathrm{Pl}}^{2}}{2} R,
$$

where $X=-g^{\mu \nu} \partial_{\mu} \phi \partial_{\nu} \phi / 2$. The general background and perturbation equations in this theory are summarized in the appendix.

\section{A. The Lagrangian for the NEC-violating phase}

Before investigating the full Lagrangian that is capable of describing the entire cosmological evolution sketched in the previous section, let us focus on the Lagrangian just for the NEC-violating phase. The Lagrangian we consider for the moment is given by

$$
\mathcal{L}=\frac{M_{\mathrm{Pl}}^{2}}{2} R+\beta X-V(\phi)-\frac{\alpha M_{\mathrm{Pl}}}{U(\phi)} X \square \phi,
$$

where

$$
V(\phi)=U(\phi)=U_{0} e^{2 \phi / M_{\mathrm{P} 1}}, \quad U_{0}=\text { const }>0,
$$

and $\alpha$ and $\beta$ are dimensionless parameters. In the Horndeski language, this is obtained by taking $G_{2}=$ $\beta X-V(\phi)$ and $G_{3}=\alpha M_{\mathrm{Pl}} X / U(\phi)$ in Eq. (3).

It is straightforward to see from the field equations (A1)-(A3) that the Lagrangian (4) with (5) admits the desired NEC-violating solution of the form

$$
H=\frac{p}{-t}, \quad \phi=M_{\mathrm{Pl}} \ln \left[\frac{M_{\mathrm{Pl}}}{\sqrt{q U_{0}}(-t)}\right],
$$

where $p>0$ and $q>0$ are dimensionless parameters characterizing the solution. These parameters are determined solely from $\alpha$ and $\beta$ : $p=p(\alpha, \beta), q=q(\alpha, \beta)$. Compact expressions are obtained if one instead writes $\alpha$ and $\beta$ in terms of $p$ and $q$ :

$$
\alpha=\frac{2 p}{q}-\frac{2}{q^{2}(1+3 p)}, \quad \beta=-2 p(2+3 p)+\frac{2}{q} .
$$

Figure 2 shows constant $\alpha$ and constant $\beta$ curves in the $p$ - $q$ plane. For given $(\alpha, \beta)$, one finds two sets of $(p, q)$ satisfying $p, q>0$.

In order for the NEC-violating background (6) to be stable, it is required that

$$
\begin{aligned}
& \mathcal{F}_{S}=-1+\left(-1-2 p+3 p^{2}\right) q>0, \\
& \mathcal{G}_{S}=2 p(1+3 p)^{3} q^{2}-3(1+3 p)^{2} q+3>0 .
\end{aligned}
$$

The two conditions are satisfied in the shaded region in Fig. 2. A stable NEC-violating solution can be found for $\alpha>0$ and $\beta<-10$. Note that all solutions with $p \leq 1$

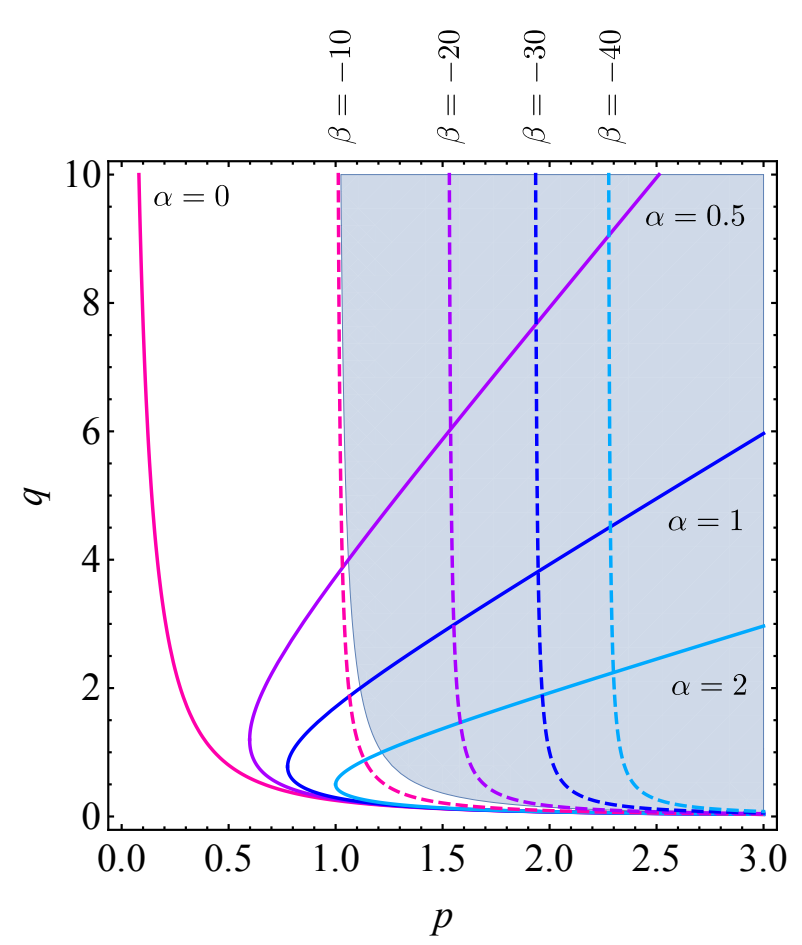

FIG. 2. Constant $\alpha$ curves (solid lines) and constant $\beta$ curves (dashed lines) in the $p$ - $q$ plane. The two stability conditions are satisfied in the shaded region.

are unstable. As we have mentioned above, we have two sets of $(p, q)$ for given $(\alpha, \beta)$. It turns out that at least one of them leads to an unstable background. (Since this unstable branch typically has $p \gg 1$ and $q \ll 1$, it is difficult to judge the stability by seeing Fig. 2.)

\section{B. The full Lagrangian}

We have thus seen that the Lagrangian (4) with (5) admits the NEC-violating solution (6), and there is a parameter region where the solution is stable against scalar perturbations. In this subsection, we modify the Lagrangian (4) so that it can be used to describe the subsequent inflation and reheating stages as well as the NEC-violating phase.

The full Lagrangian is given by

$$
\mathcal{L}=\frac{M_{\mathrm{Pl}}^{2}}{2} R+B(\phi) X-V(\phi)-\frac{\alpha M_{\mathrm{Pl}}}{U(\phi)} X \square \phi,
$$

where we now take

$$
\begin{aligned}
V(\phi) & =\frac{U(\phi) \cdot m^{2} \phi^{2}}{2 U(\phi)+m^{2} \phi^{2}} \\
B(\phi) & =\frac{1+\beta}{2}+\frac{1-\beta}{2} \tanh \left[c\left(\phi-\phi_{*}\right) / M_{\mathrm{Pl}}\right]
\end{aligned}
$$

with $U(\phi)=U_{0} e^{2 \phi / M_{\mathrm{Pl}}}$. The potential $V(\phi)$ is taken so that $V \simeq U$ for negatively large $\phi$ and $V \simeq m^{2} \phi^{2} / 2$ for 
small $\phi$, as shown in Fig. 1. Any potential with a similar form is possible as well, but we use this particular form for simplicity. The potential peaks at $\phi=\phi_{*}(<0)$, where

$$
\phi_{*}=-\frac{3}{2} M_{\mathrm{Pl}} W_{0}(z), \quad z=\frac{2^{4 / 3}}{3}\left(\frac{U_{0}}{M_{\mathrm{Pl}}^{2} m^{2}}\right)^{1 / 3},
$$

with $W_{0}$ being the Lambert $W$ function (the principal branch of the inverse functions of $\left.z=W e^{W}\right)$. The coefficient of the kinetic term, $B(\phi)$, is taken so that $B \simeq \beta(<0)$ for $\phi<\phi_{*}$ and $B \simeq 1$ for $\phi>\phi_{*}$. Here, $c$ is some constant of $\mathcal{O}(1)-\mathcal{O}(10)$ whose explicit value is not important. The sign flip of the kinetic term soon after the end of the NEC-violating phase is necessary for the subsequent inflation and reheating stages to be free from ghost instability. This idea is analogous to what is supposed to happen at the end of k-inflation [68].

The Lagrangian (10) reduces to Eq. (4) with (5) for negatively large $\phi$, giving rise to the NEC-violating solution (6). The sign of the kinetic term is flipped when $\phi$ goes over the top of the potential, and inflation then starts in the potential $V \simeq m^{2} \phi^{2} / 2$ provided that $\left|\phi_{*}\right| \gtrsim$ $M_{\mathrm{Pl}}$. After inflation, reheating occurs in a usual way at the bottom of the $\phi^{2}$ potential. We thus expect that the scenario outlined in the previous section is realized by the Lagrangian (10). This will be demonstrated explicitly by numerical calculations in the next subsection.

We have four key quantities characterizing our model: $p$ in the expansion law in the NEC-violating phase, the maximum value of the Hubble parameter reached at the end of the NEC-violating phase (denoted by $H_{*}$ ), the inflationary Hubble scale (hereafter denoted by $H_{\text {inf }}$ ), and the duration of inflation (hereafter denoted by $\Delta \mathcal{N}_{\text {inf }}$ ). These quantities are determined by the four parameters in the Lagrangian $(10)\left(\alpha, \beta, U_{0}\right.$, and $\left.m\right) .{ }^{1}$ As we have already seen, $p$ is determined by $\alpha$ and $\beta$ through the relation (7). Assuming that the transition from the NECviolating phase to inflation is sudden, one can estimate as

$$
\begin{aligned}
H_{*} & \sim \frac{p \sqrt{q U_{0}}}{M_{\mathrm{Pl}}} e^{\phi_{*} / M_{\mathrm{Pl}}}=p \sqrt{\frac{q}{2} m\left(\frac{\left|\phi_{*}\right|}{M_{\mathrm{Pl}}}\right)^{3 / 2},} \\
H_{\mathrm{inf}} & \sim \frac{m\left|\phi_{*}\right|}{\sqrt{6} M_{\mathrm{Pl}}}
\end{aligned}
$$

where $\phi_{*}=\phi_{*}\left(U_{0}, m\right)$ was already defined in Eq. (13). In deriving the estimate (14), we used $H_{*} \sim p /\left(-t_{*}\right)$ and $\phi_{*} \sim M_{\mathrm{Pl}} \ln \left[M_{\mathrm{Pl}} / \sqrt{q U_{0}}\left(-t_{*}\right)\right]$, where $t_{*}$ is the time at the end of the NEC-violating phase. Finally, a simple textbook calculation yields

$$
\Delta \mathcal{N}_{\text {inf }} \sim \frac{1}{4}\left(\frac{\left|\phi_{*}\right|}{M_{\mathrm{Pl}}}\right)^{2}
$$

\footnotetext{
1 The parameter $c$ plays essentially no role in the dynamics.
}

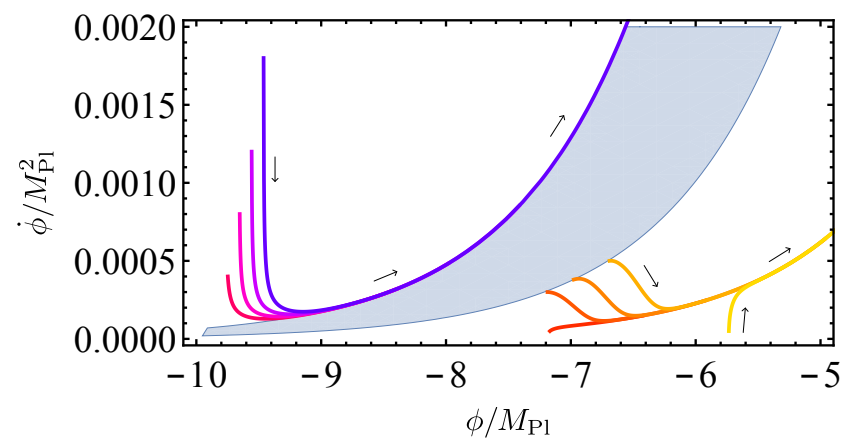

FIG. 3. Typical phase-space trajectories. The Friedmann constraint does not have real roots for $H$ in the shaded region.

As seen from Eq. (16), we can adjust the number of e-folds of inflation by shifting the top of the potential $\phi_{*}$. To demonstrate how $\phi_{*}$ depends on $U_{0}$, it is convenient to introduce a new parameter $\phi_{0}$ defined as $\phi_{0}:=(1 / 2) M_{\mathrm{Pl}} \ln \left(U_{0} / M_{\mathrm{Pl}}^{4}\right)$ (i.e., $\left.U_{0}=M_{\mathrm{Pl}}^{4} e^{2 \phi_{0} / M_{\mathrm{Pl}}}\right)$. The shift $\phi_{0} \rightarrow \phi_{0}+c$ with $c>0(c<0)$ causes left (right) shifting of $U(\phi)$. The Lambert $W$ function $W_{0}(z)$ can be approximated roughly by $W_{0}(z) \approx \ln (z / \ln z)$ or more crudely $W_{0}(z) \sim \ln z$ for large $z>0$. Using the latter approximation here in Eq. (13), we get $\phi_{*} \sim-\phi_{0}+$ const. We typically take $\phi_{0} / M_{\mathrm{Pl}} \sim \mathcal{O}(10)$ to sustain a sufficient duration of inflation. One might suspect that such a value of $\phi_{0}$ yielded extremely large $U_{0}$ and the universe experienced super-Planck scale $H$. However, since $M_{\mathrm{Pl}}^{2} H^{2} \lesssim V\left(\phi_{*}\right)=(1 / 2) m^{2} \phi_{*}^{3} /\left(\phi_{*}-M_{\mathrm{Pl}}\right)$, it is not the case as long as $\left|\phi_{*}\right| \ll M_{\mathrm{Pl}}^{2} / m$. Hereafter we use $\phi_{0}$ instead of $U_{0}$.

\section{Numerical results}

Let us first affirm that the NEC-violating solution (6) is an attractor. Figure 3 shows the behavior of the solutions for $(\alpha, \beta)=(1.09,-12.4)$ with different initial conditions, obtained by solving the dynamical equations (A2) and (A3) numerically. ${ }^{2}$ We see that the trajectories converge to either of the two curves, $\dot{\phi} \propto$ $\sqrt{q} e^{\phi / M_{\mathrm{P} 1}}$, with different $q$. In the upper left region the solutions converge to the stable NEC-violating solution with $(p, q) \simeq(1.2,2)$, while in the lower right region the solutions converge to the unstable background with $(p, q) \simeq\left(6.1,8.4 \times 10^{-3}\right)$. The two regions of initial conditions are separated by the "no-go" region (the shaded region in Fig. 3) where the Friedmann constraint equation does not have real roots for $H$. In the following analysis, we assume that the initial conditions for $(\phi, \dot{\phi})$

\footnotetext{
2 The initial value for $H$ is determined from the Friedmann constraint (A1). We check that the constraint (A1) is satisfied at each time step.
} 


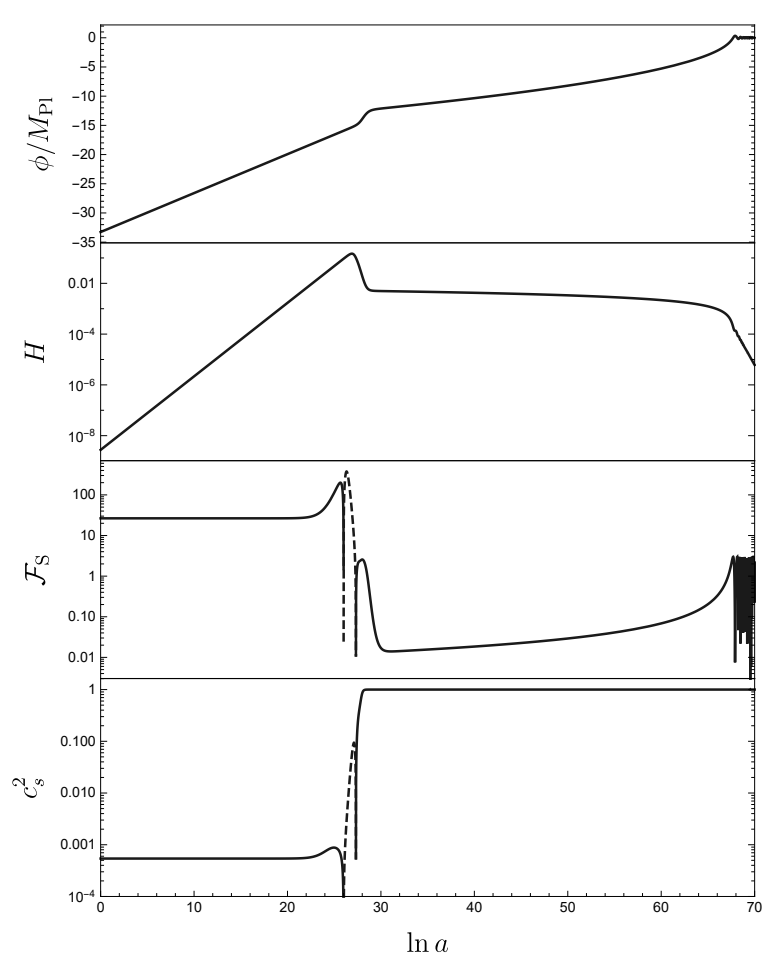

FIG. 4. Time evolution of $\phi, H, \mathcal{F}_{S}$, and $c_{s}^{2}\left(=\mathcal{F}_{S} / \mathcal{G}_{S}\right)$. The model parameters are chosen as $p=3 / 2\left(n_{t}=0.8\right)$, $q=10, m^{2}=10^{-6} M_{\mathrm{Pl}}^{2}$, and $\phi_{0}=12 M_{\mathrm{Pl}}$. In the plots, the dashed lines denote the negative value having the same absolute value.

are in the upper left region so that they lead to a stable NEC-violating universe.

We then investigate the entire evolution numerically. In Figure 4, we plot the time evolution of $\phi, H, \mathcal{F}_{S}$, and $c_{s}^{2}:=\mathcal{F}_{S} / \mathcal{G}_{S}$ for the parameter choice $p=3 / 2$ $\left(n_{t}=0.8\right), q=10, m^{2}=10^{-6} M_{\mathrm{Pl}}^{2}$, and $\phi_{0}=12 M_{\mathrm{Pl}}$. In the NEC-violating phase $(0<\ln a<26.0)$, we have positive $\mathcal{F}_{S}$. Note that $H_{*}$ is as high as $\mathcal{O}(0.1) \times M_{\mathrm{Pl}}$. In the short decelerating phase $(26.0<\ln a<27.3), \mathcal{F}_{S}$ becomes negative, which does not contradict the stability condition (8) since the system is no longer on the attractor (6). This point will be discussed shortly. In the inflationary phase $(27.3<\ln a \lesssim 67), \mathcal{F}_{S}$ is approximately given by $-\mathrm{d} \ln H / \mathrm{d} \ln a$, which is positive in an NEC-preserving universe. At $\ln a=67.7, \phi$ passes the origin and starts to oscillate, leading to the expansion of a matter-dominated universe.

Our numerical result implies a potential drawback of the present model that it exhibits a gradient instability in the short intermediate stage between the NEC-violating and inflationary phases. This is similar to what happens at the transition from the galilean genesis phase to inflation [46], and in fact the appearance of gradient instabilities is generic to non-singular cosmological solutions in galileon/Horndeski theories [69, 70]. In the present case, however, the instability could in principle be avoidable by more elaborate choices of the Lagrangian and model parameters, because the background (1) is singular in the $t \rightarrow-\infty$ limit $^{3}$ and hence the "no-go" theorems in $[69,70]$ do not apply. The instability issue can also be resolved by introducing "beyond-Horndeski" or higherorder operators [48, 71-73].

\section{GRAVITATIONAL WAVE SPECTRUM}

Since we do not consider the scalar field derivatively coupled to the curvature through the so-called Horndeski $G_{4}(\phi, X)$ and $G_{5}(\phi, X)$ terms, the tensor perturbations $h_{i j}$ evolve following the same equation as in general relativity. Their equation of motion is

$$
h_{i j}^{\prime \prime}+2\left(a^{\prime} / a\right) h_{i j}^{\prime}-\partial^{2} h_{i j}=0
$$

where a prime denotes the derivative with respect to the conformal time $\eta$. We move to Fourier space, expand $h_{i j}$ into the basis of the polarization tensors, and write

$$
h_{i j}(\eta, \vec{x})=\frac{1}{(2 \pi)^{3}} \sum_{A=+, \times} \int \mathrm{d}^{3} k \tilde{h}_{A}(\eta, \vec{k}) e_{i j}^{A}(\vec{k}) e^{i \vec{k} \cdot \vec{x}}
$$

where $e_{i j}^{A}$ is transverse, traceless, and normalized as $e_{i j}^{A} e_{i j}^{A^{\prime}}=\delta_{A^{\prime}}^{A}$. Following the usual quantization procedure, we obtain the canonically normalized positive frequency mode in the NEC-violating phase as

$$
\tilde{h}_{A}=\frac{\sqrt{\pi}}{a M_{\mathrm{Pl}}} \sqrt{-\eta} H_{\nu}^{(1)}(-k \eta)
$$

where $H_{\nu}^{(1)}$ is the Hankel function and $\nu:=(3 p+1) / 2(p+$ $1)$. The initial conditions for the tensor perturbations are set by Eq. (19). In the superhorizon regime, $|k \eta| \ll 1$, we have

$$
k^{3}\left|\tilde{h}_{A}\right|^{2} \simeq \frac{2^{2 \nu} \Gamma^{2}(\nu)}{\pi M_{\mathrm{Pl}}^{2}}\left(\frac{2 H_{c}}{2 \nu-1}\right)^{2 \nu-1} k^{3-2 \nu}=\mathrm{const},
$$

where we introduced the constant $H_{c}:=\mathrm{Ha}^{-1 / p}$. From this, we get the primordial power spectrum of gravitational waves as

$$
\mathcal{P}_{\mathrm{GW}}=2 \cdot \frac{k^{3}}{2 \pi^{2}}\left|\tilde{h}_{A}\right|^{2}=\frac{2^{2 \nu} \Gamma(\nu)^{2}}{\pi^{3} M_{\mathrm{Pl}}^{2}}\left(\frac{2 H_{c}}{2 \nu-1}\right)^{2 \nu-1} k^{3-2 \nu},
$$

Note that for $\nu=3 / 2$ this reproduces the usual primordial power spectrum in de Sitter inflation. From Eq. (21)

\footnotetext{
3 Since $\int_{-\infty}^{t} a \mathrm{~d} t<\infty$ for $p>1$, this NEC-violating universe is past incomplete. In this sense, the present model is as bad as inflation.
} 


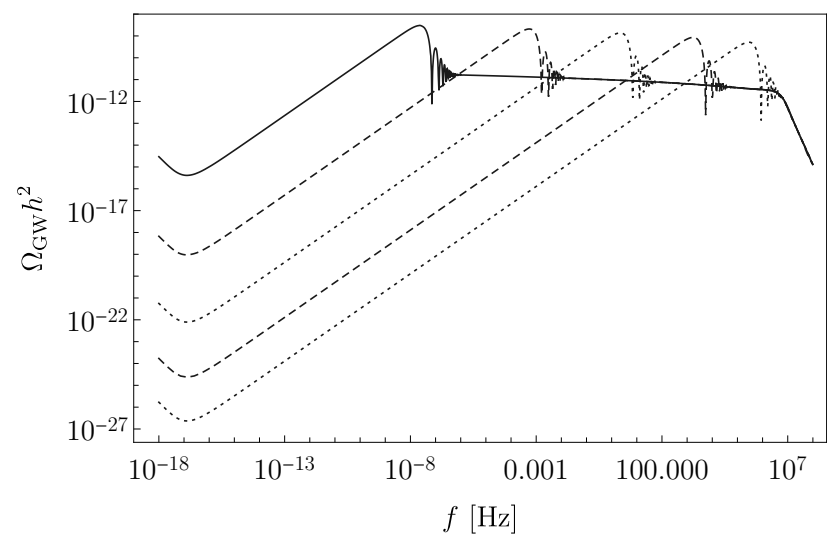

FIG. 5. Gravitational wave spectra in the cases of $\phi_{0} / M_{\mathrm{Pl}}=$ 12 (solid), 10 (left dashed), 8 (left dotted), 6 (right dashed), and 4 (right dotted). The other parameters are the same as in Fig. 4.

we see that the modes which exit the horizon in the NECviolating phase have a primordial spectrum with a blue spectral tilt (2), whereas those which exit the horizon in the subsequent inflationary phase have an almost flat primordial spectrum with $n_{t}=-2 \epsilon(<0)$, where $\epsilon$ is the usual small slow-roll parameter, $\epsilon:=-\dot{H} / H^{2}$. After re-entering the horizon their amplitudes decay proportionally to $1 / a$.

The present energy density spectrum of gravitational waves is given by

$$
\Omega_{\mathrm{GW}}(k)=\frac{k^{5}\left(\left|\tilde{h}_{+}\right|^{2}+\left|\tilde{h}_{\times}\right|^{2}\right)}{24 \pi^{2} a^{2} H_{0}^{2}},
$$

and below we show $\Omega_{\mathrm{GW}}(f)$ plots, where $k$ is the comoving wavenumber, $f$ is the physical frequency $f=k / 2 \pi a$, and $H_{0}$ is the Hubble constant. Each spectrum in our model is typically composed by the three parts; an extremely blue-tilted spectrum in the low frequency range; wiggles in the middle frequency range (due to the intermediate decelerating stage); and a slightly red-tilted spectrum in the high frequency range. We plot the energy density spectra for different values of $\phi_{0}$ in Fig. 5 . A larger value of $\phi_{0}$ leads to a larger number of e-folds, a lower peak frequency, and as a result larger $\Omega_{\mathrm{GW}}(f)$ on CMB scales. Note in passing that here and hereafter we assume that the Hubble parameter at which the reheating process becomes so efficient that the energy is converted from matter to radiation rapidly, $H_{\mathrm{R}}$, is given by $H_{\mathrm{R}}=10^{-8} M_{\mathrm{Pl}}$.

Let us study the effects of changing the other parameters $m, q$, and $p$. Since we have $\left|\phi_{*}\right| / M_{\mathrm{Pl}} \sim \mathcal{O}(1-10), m$ is the only parameter which determines the order of an energy scale of the slow-rolling phase, although $\phi_{*}$ also has an implicit logarithmic dependence on $m$ and $\phi_{0}$. We plot the energy density spectra for different $m$ in Fig. 6 . From Eq. (14), once we set $q m^{2}$ constant, we get simi-

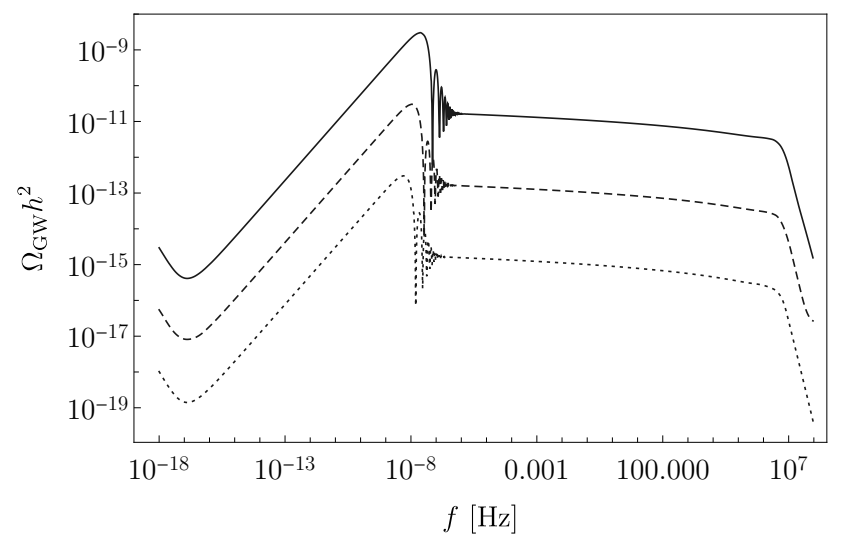

FIG. 6. Gravitational wave spectra in the cases of $m^{2} / M_{\mathrm{Pl}}^{2}=$ $10^{-6}$ (solid), $10^{-8}$ (dashed), and $10^{-10}$ (dotted). In each spectrum, we set $\phi_{0} / M_{\mathrm{Pl}}=12+\ln \left(10^{3} \mathrm{~m} / M_{\mathrm{Pl}}\right)$ to have nearly constant $\phi_{*}$. The other parameters are the same as in Fig. 4 .

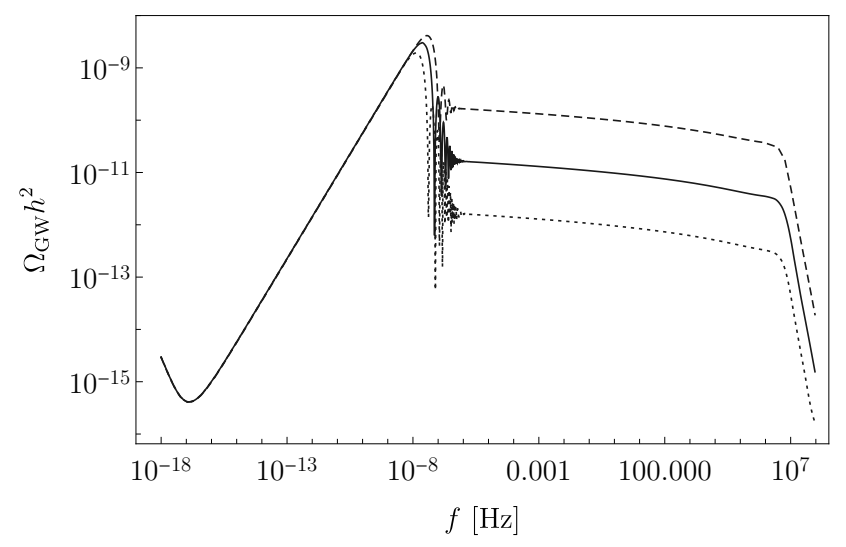

FIG. 7. Gravitational wave spectra in the cases of $m^{2}=10^{-5}$ (dashed), $10^{-6}$ (solid), and $10^{-7}$ (dotted). The parameter $q$ are taken as $q m^{2}=10^{-5}$ in order to produce similar bluetilted spectra. The other parameters are the same as in Fig. 4.

lar values of $H_{*}$ even with different $m$. We show energy density spectra in the case of different $m$ but the same $q m^{2}$ in Fig. 7.

To compare our results with the NANOGrav observation [1], we parametrize the characteristic strain of gravitational waves around the reference frequency $f_{\mathrm{yr}}=1 / \mathrm{yr}$ as

$$
h_{c}(f)=A_{\mathrm{GW}}\left(\frac{f}{f_{\mathrm{yr}}}\right)^{\alpha}
$$

which is related to $\Omega_{\mathrm{GW}}$ as $\Omega_{\mathrm{GW}}=2 \pi^{2} f^{2} h_{c}^{2} /\left(3 H_{0}^{2}\right)$. The relation between $\alpha$ and the slope of the crosspower spectral density $\gamma=3-2 \alpha$ is also useful for direct comparison to the observational implication. Since $\Omega_{\mathrm{GW}}(f) \propto f^{n_{t}}$ is observed for the modes which reenter the horizon in the radiation-dominated era, we get 
$\alpha=\left(n_{t}-2\right) / 2=-p /(p+1)$ from Eq. (2), if the extremely blue-tilted gravitational waves from the NECviolating phase give rise to the spectrum around $f_{\mathrm{yr}}$. In Fig. 8, we plot the energy density spectra for different values of $p$. We also show the sensitivity curves for present and future experiments of pulsar timing arrays and space/ground-based interferometers. For the plotted spectra, their spectral slopes and amplitudes are $\left(\gamma, \log _{10} A_{\mathrm{GW}}\right)=(4.2,-14.525),(4.1,-14.542)$, and $(4.05,-14.535)$, for $n_{t}=0.8,0.9$, and 0.95 , respectively, which are within the $2-\sigma$ posterior contour for the five frequency power-law model of a spatially-uncorrelated common-spectrum process [1].

Finally, let us discuss the BBN constraint [23, 76-79] in our model. We calculate the total energy density of gravitational waves for the case with $n_{t}=0.8$ in Fig. 8 and obtain its contribution to the effective number of relativistic species $\Delta N_{\text {eff }}$ as

$$
\Delta N_{\mathrm{eff}} \simeq \int \mathrm{d} \ln f \frac{\Omega_{\mathrm{GW}} h^{2}}{5.6 \times 10^{-6}} \simeq 1.3 \times 10^{-3} .
$$

This is much smaller than the upper bound from the BBN constraint, $\Delta N_{\text {eff }}<\mathcal{O}(0.1)[80,81]$. (The similar bound also comes from the large scale observations [82, 83].) Since we have the nearly flat part of the spectrum in the high-frequency range, we can avoid the problem pointed out by [20], in which a single power-law, blue-tilted spectrum violates the BBN constraint if it is consistent with both the CMB constraint and the NANOGrav result.

\section{SCALAR PERTURBATIONS}

Large-scale curvature perturbations must be generated in the early universe to account for the observed nearly scale-invariant spectrum of fluctuations. In our scenario, however, it cannot be due to the fluctuations of the $\phi$ field, because in the NEC-violating phase we have $\mathcal{G}_{S}=$ const and $\mathcal{F}_{S}=$ const, which means that the curvature perturbation $\zeta$ has a highly blue spectrum with $n_{s}-1=$ $n_{t}>0$ and hence is irrelevant on CMB scales.

To generate nearly scale-invariant curvature perturbations on CMB scales, we need another scalar field. Since the background in the NEC-violating phase is significantly away from de Sitter, a minimally coupled scalar field does not give rise to a scale-invariant spectrum of fluctuations. We therefore extend the mechanism proposed originally in the context of the galilean genesis scenario [38] to a more general background, and consider a non-minimally coupled scalar field,

$$
S_{\sigma}=\int \mathrm{d}^{4} x \sqrt{-\widetilde{g}}\left[-\frac{1}{2} \widetilde{g}^{\mu \nu} \partial_{\mu} \sigma \partial_{\nu} \sigma-\mathcal{V}(\sigma)\right],
$$

with

$$
\widetilde{g}_{\mu \nu}=e^{2 \phi / M_{\mathrm{Pl}}} g_{\mu \nu}
$$

The conformally related metric (26) reads more explicitly

$$
\widetilde{g}_{\mu \nu} \mathrm{d} x^{\mu} \mathrm{d} x^{\nu}=\widetilde{a}^{2}\left(-\mathrm{d} \eta^{2}+\mathrm{d} \vec{x}^{2}\right),
$$

where

$$
\widetilde{a}=e^{\phi / M_{\mathrm{Pl}}} a \propto \frac{1}{(-\eta)},
$$

in the NEC-violating phase, irrespective of the value of $p$. This means that the non-minimally coupled scalar field $\sigma$ effectively lives in de Sitter, generating a nearly scale-invariant spectrum. We therefore expect that fluctuations of the $\sigma$ field are the origin of the observed spectrum of scalar perturbations. To obtain a more detailed prediction, we need to investigate the process of converting the fluctuations of $\sigma$ to adiabatic modes, which is model-dependent and deserves further study.

\section{CONCLUSIONS}

In this paper, we have explored the possibility to generate nanohertz gravitational waves consistent with both of the CMB and BBN constraints. We investigated the scenario which results in an extremely blue-tilted spectrum appropriate for the $\mathrm{CMB}$ constraint at $f \lesssim 10^{-15} \mathrm{~Hz}$ and the NANOGrav result at $f \sim 1 / \mathrm{yr}$ and a (nearly) flat spectrum for $f \gtrsim 1 / \mathrm{yr}$ to evade the BBN constraint. To produce an extremely blue spectrum, we considered an NEC-violating phase in the early universe undergoing the (super-)accelerated expansion with $a \propto(-t)^{p}$ $(p>0)$, in which the tensor spectral index $n_{t}>0$ is related to the power-law index $p$ as in Eq. (2). To obtain a flat spectrum in a high frequency range, we examined the scenario in which slow-roll inflation follows after the NEC-violating phase and ends with standard reheating, as sketched out in the potential of Fig. 1.

In Sec. III, we proposed a successful model in the cubic Horndeski theory. First, we gave the minimal action (4) which can cause a stable NEC-violating phase. We obtained the relation between the model parameters $(\alpha, \beta)$ and the solution parameters $(p, q)$ in Eq. (7), and the stability condition in terms of $(p, q)$ in Eqs. (8) and (9). From these conditions, we found that a solution with $p \leq 1$ is always unstable within our model, while stable solutions with $p>1$ are indeed possible. We expect that a stable model with $p \leq 1$ can be realized by adding other higher-order terms in the action, but the analysis would be more complicated. Next, we assume that the potential and the coefficient of the kinetic term are given by Eq. (12), which changes the field dynamics for $\phi>\phi_{*}$, connecting the NEC-violating phase to the subsequent inflationary phase. The phenomenological parameters $\left(H_{*}, H_{\text {inf }}, \Delta \mathcal{N}_{\text {inf }}\right)$ are estimated in Eqs. (14), (15), and (16), enabling us to design the gravitational wave spectrum. In Fig. 4, we numerically gave the typical time evolution of the background and showed that a gradient instability occurs in the intermediate decelerating 


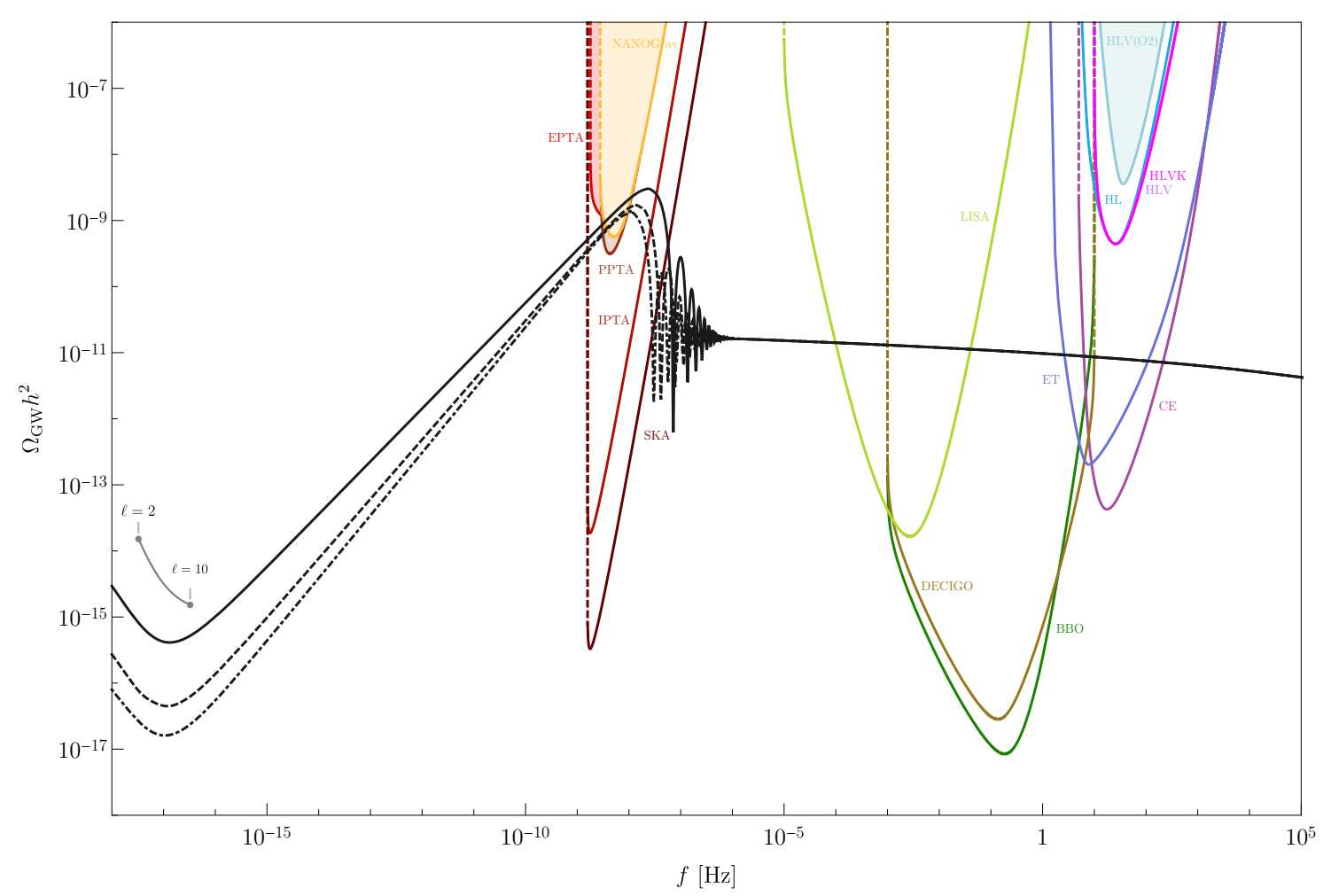

FIG. 8. Energy density spectra of gravitational waves in the cases of $n_{t}=0.8$ (solid), 0.9 (dashed), and 0.95 (dot-dashed), which correspond to $p=3 / 2,11 / 9$, and $21 / 19$, respectively. The other parameters are the same as in Fig. 4 . We give the power-law-integrated sensitivity curves [74] for present and future experiments of pulsar-timing arrays and space/ground-based interferometers. For comparison with the lower-frequency constraint, we also plot as the gray line the spectrum with $n_{t}=0$ and $r=0.07$, the upper limit from the BICEP2/Keck Array and Planck experiments [75], between the frequencies corresponding to multipoles $\ell=2$ and 10 .

phase. We have discussed that this issue can be resolved by higher-order operators such as in beyond-Horndeski theories [48, 71-73].

In Sec. IV, we have presented the gravitational wave spectrum (22) in our model with different parameter choices. As is expected, those spectra are composed of the blue-tilted part and the nearly flat part, of which amplitudes can be controlled almost independently of each other. We gave gravitational wave spectra with $n_{t}=0.8$, 0.9 , and 0.95 for $f \lesssim 1 /$ yr in Fig. 8 , which are consistent with the recent NANOGrav result [1]. We found that thanks to the flat part of the spectrum we are no longer bothered by the BBN bound and this flat part is possibly detected by future space/ground-based observations. In Sec. V, we discussed how the nearly scale-invariant scalar perturbations observed by CMB experiments are produced by introducing another non-minimally coupled scalar field (10) which effectively lives in de Sitter even in the NEC-violating phase.

In summary, we have obtained an explicit early universe model that can yield an extremely blue gravitational wave spectrum peaking at $f \sim 1$ /yr explored by pulsar timing arrays, while being consistent with other observational constraints.

\section{ACKNOWLEDGMENTS}

We thank Kohei Kamada and Sachiko Kuroyanagi for useful discussions. HWHT was supported by the Grant-in-Aid for JSPS Research Fellow No. JP19J11640. TK was partially supported by JSPS KAKENHI Grant Nos. JP20H04745 and JP20K03936.

\section{Appendix A: General background and perturbation equations for the cubic Horndeski theory}

We replicate the background and perturbation equations for cubic Horndeski cosmology based on the Lagrangian (3). The results are well known in the literature (see Refs. [33, 35, 36]).

The background equations for the metric $\mathrm{d} s^{2}=-\mathrm{d} t^{2}+$ $a^{2}(t) \mathrm{d} \vec{x}^{2}$ and the scalar field $\phi=\phi(t)$ are given by

$$
\begin{aligned}
& 3 M_{\mathrm{Pl}}^{2} H^{2}=2 X G_{2 X}-G_{2}+6 H X \dot{\phi} G_{3 X}-2 X G_{3 \phi}, \quad(\mathrm{A} 1) \\
& -M_{\mathrm{Pl}}^{2} \dot{H}=X G_{2 X}+3 H X \dot{\phi} G_{3 X}-X\left(2 G_{3 \phi}+\ddot{\phi} G_{3 X}\right),
\end{aligned}
$$




$$
\begin{aligned}
& \frac{1}{a^{3}} \frac{\mathrm{d}}{\mathrm{d} t}\left[a^{3}\left(\dot{\phi} G_{2 X}+6 H X G_{3 X}-2 \dot{\phi} G_{3 \phi}\right)\right] \\
& =G_{2 \phi}-2 X\left(G_{3 \phi \phi}+\ddot{\phi} G_{3 \phi X}\right) .
\end{aligned}
$$

Since $\phi$ is minimally coupled to the curvature tensor, the equation for tensor modes, $h_{i j}(t, \vec{x})$, is the same as the standard one known in general relativity. The quadratic action for the curvature perturbation in the unitary gauge, $\zeta(t, \vec{x})$, is given by

$$
S_{\zeta}^{(2)}=M_{\mathrm{Pl}}^{2} \int \mathrm{d} t \mathrm{~d}^{3} x a^{3}\left[\mathcal{G}_{S} \dot{\zeta}^{2}-\frac{\mathcal{F}_{S}}{a^{2}}(\partial \zeta)^{2}\right]
$$

where

$$
\mathcal{G}_{S}=\frac{1}{a} \frac{\mathrm{d}}{\mathrm{d} t}\left(\frac{a}{H-\dot{\phi} X G_{3 X} / M_{\mathrm{Pl}}^{2}}\right)-1
$$

$$
\mathcal{F}_{S}=\frac{\Sigma}{\left(H-\dot{\phi} X G_{3 X} / M_{\mathrm{Pl}}^{2}\right)^{2}},
$$

with

$$
\begin{aligned}
M_{\mathrm{Pl}}^{2} \Sigma= & X G_{2 X}+2 X^{2} G_{2 X X}+6 H \dot{\phi} X G_{3 X} \\
& +6 H \dot{\phi} X^{2} G_{3 X X}-2 X G_{3 \phi}-2 X^{2} G_{3 \phi X} \\
& +6 X^{3} G_{3 X}^{2} / M_{\mathrm{Pl}}^{2} .
\end{aligned}
$$

The background under consideration is stable if $\mathcal{G}_{S}>0$ and $c_{s}^{2}:=\mathcal{F}_{S} / \mathcal{G}_{S}>0$.
[1] NANOGrav collaboration, Z. Arzoumanian et al., The NANOGrav 12.5-year Data Set: Search For An Isotropic Stochastic Gravitational-Wave Background, 2009.04496.

[2] R. Hellings and G. Downs, UPPER LIMITS ON THE ISOTROPIC GRAVITATIONAL RADIATION BACKGROUND FROM PULSAR TIMING ANALYSIS, Astrophys. J. Lett. 265 (1983) L39.

[3] J. Ellis and M. Lewicki, Cosmic String Interpretation of NANOGrav Pulsar Timing Data, 2009.06555.

[4] S. Blasi, V. Brdar and K. Schmitz, Has NANOGrav found first evidence for cosmic strings?, 2009.06607.

[5] W. Buchmuller, V. Domcke and K. Schmitz, From NANOGrav to LIGO with metastable cosmic strings, 2009.10649.

[6] R. Samanta and S. Datta, Gravitational wave complementarity and impact of NANOGrav data on gravitational leptogenesis: cosmic strings, 2009.13452.

[7] Y. Nakai, M. Suzuki, F. Takahashi and M. Yamada, Gravitational Waves and Dark Radiation from Dark Phase Transition: Connecting NANOGrav Pulsar Timing Data and Hubble Tension, 2009.09754.

[8] A. Addazi, Y.-F. Cai, Q. Gan, A. Marciano and K. Zeng, NANOGrav results and Dark First Order Phase Transitions, 2009.10327.

[9] W. Ratzinger and P. Schwaller, Whispers from the dark side: Confronting light new physics with NANOGrav data, 2009.11875.

[10] A. Neronov, A. Roper Pol, C. Caprini and D. Semikoz, NANOGrav signal from MHD turbulence at QCD phase transition in the early universe, 2009.14174.

[11] K. T. Abe, Y. Tada and I. Ueda, Induced gravitational waves as a cosmological probe of the sound speed during the QCD phase transition, 2010.06193.

[12] V. Vaskonen and H. Veermäe, Did NANOGrav see a signal from primordial black hole formation?, 2009.07832.

[13] V. De Luca, G. Franciolini and A. Riotto, NANOGrav Hints to Primordial Black Holes as Dark Matter, 2009.08268.

[14] K. Kohri and T. Terada, Solar-Mass Primordial Black
Holes Explain NANOGrav Hint of Gravitational Waves, 2009.11853.

[15] G. Domènech and S. Pi, NANOGrav Hints on Planet-Mass Primordial Black Holes, 2010.03976.

[16] S. Bhattacharya, S. Mohanty and P. Parashari, Implications of the NANOGrav result on primordial gravitational waves in nonstandard cosmologies, 2010.05071.

[17] R. Namba and M. Suzuki, Implications of Gravitational-wave Production from Dark Photon Resonance to Pulsar-timing Observations and Effective Number of Relativistic Species, 2009.13909.

[18] N. Kitajima, J. Soda and Y. Urakawa, Nano-Hz gravitational wave signature from axion dark matter, 2010.10990.

[19] L. Bian, J. Liu and R. Zhou, NanoGrav 12.5-yr data and different stochastic Gravitational wave background sources, 2009.13893.

[20] S. Vagnozzi, Implications of the NANOGrav pulsar timing results for inflation, 2009.13432.

[21] H.-H. Li, G. Ye and Y.-S. Piao, Is the NANOGrav signal a hint of dS decay during inflation?, 2009.14663.

[22] M. Guzzetti, N. Bartolo, M. Liguori and S. Matarrese, Gravitational waves from inflation, Riv. Nuovo Cim. 39 (2016) 399 [1605.01615].

[23] S. Kuroyanagi, T. Takahashi and S. Yokoyama, Blue-tilted Tensor Spectrum and Thermal History of the Universe, JCAP 02 (2015) 003 [1407.4785].

[24] Y.-S. Piao and E. Zhou, Nearly scale invariant spectrum of adiabatic fluctuations may be from a very slowly expanding phase of the universe, Phys. Rev. D 68 (2003) 083515 [hep-th/0308080].

[25] Y.-S. Piao and Y.-Z. Zhang, Phantom inflation and primordial perturbation spectrum, Phys. Rev. D 70 (2004) 063513 [astro-ph/0401231].

[26] Y.-S. Piao and Y.-Z. Zhang, The Primordial perturbation spectrum from various expanding and contracting phases, Phys. Rev. D 70 (2004) 043516 [astro-ph/0403671].

[27] Y.-S. Piao, Gravitational wave background from phantom superinflation, Phys. Rev. D 73 (2006) 047302 
[gr-qc/0601115].

[28] Y.-S. Piao, Primordial perturbations during a slow expansion, Phys. Rev. D 76 (2007) 083505 [0706.0981].

[29] M. Baldi, F. Finelli and S. Matarrese, Inflation with violation of the null energy condition, Phys. Rev. D 72 (2005) 083504 [astro-ph/0505552].

[30] P. Creminelli, M. A. Luty, A. Nicolis and L. Senatore, Starting the Universe: Stable Violation of the Null Energy Condition and Non-standard Cosmologies, JHEP 12 (2006) 080 [hep-th/0606090].

[31] G. W. Horndeski, Second-order scalar-tensor field equations in a four-dimensional space, Int. J. Theor. Phys. 10 (1974) 363.

[32] C. Deffayet, X. Gao, D. Steer and G. Zahariade, From $k$-essence to generalised Galileons, Phys. Rev. D 84 (2011) 064039 [1103.3260].

[33] T. Kobayashi, M. Yamaguchi and J. Yokoyama, Generalized G-inflation: Inflation with the most general second-order field equations, Prog. Theor. Phys. 126 (2011) 511 [1105.5723].

[34] T. Kobayashi, Horndeski theory and beyond: a review, Rept. Prog. Phys. 82 (2019) 086901 [1901.07183].

[35] C. Deffayet, O. Pujolas, I. Sawicki and A. Vikman, Imperfect Dark Energy from Kinetic Gravity Braiding, JCAP 10 (2010) 026 [1008.0048].

[36] T. Kobayashi, M. Yamaguchi and J. Yokoyama, G-inflation: Inflation driven by the Galileon field, Phys. Rev. Lett. 105 (2010) 231302 [1008.0603].

[37] A. Anisimov, E. Babichev and A. Vikman, B-inflation, JCAP 06 (2005) 006 [astro-ph/0504560].

[38] P. Creminelli, A. Nicolis and E. Trincherini, Galilean Genesis: An Alternative to inflation, JCAP 11 (2010) 021 [1007.0027].

[39] Z.-G. Liu, J. Zhang and Y.-S. Piao, A Galileon Design of Slow Expansion, Phys. Rev. D 84 (2011) 063508 [1105.5713].

[40] Z.-G. Liu and Y.-S. Piao, A Galileon Design of Slow Expansion: Emergent universe, Phys. Lett. B 718 (2013) 734 [1207.2568].

[41] P. Creminelli, K. Hinterbichler, J. Khoury, A. Nicolis and E. Trincherini, Subluminal Galilean Genesis, JHEP 02 (2013) 006 [1209.3768].

[42] K. Hinterbichler, A. Joyce, J. Khoury and G. E. Miller, DBI Realizations of the Pseudo-Conformal Universe and Galilean Genesis Scenarios, JCAP 12 (2012) 030 [1209.5742].

[43] K. Hinterbichler, A. Joyce, J. Khoury and G. E. Miller, Dirac-Born-Infeld Genesis: An Improved Violation of the Null Energy Condition, Phys. Rev. Lett. 110 (2013) 241303 [1212.3607].

[44] Z.-G. Liu and Y.-S. Piao, Galilean Islands in Eternally Inflating Background, Phys. Rev. D 88 (2013) 043520 [1301.6833].

[45] B. Elder, A. Joyce and J. Khoury, From Satisfying to Violating the Null Energy Condition, Phys. Rev. D 89 (2014) 044027 [1311.5889].

[46] D. Pirtskhalava, L. Santoni, E. Trincherini and P. Uttayarat, Inflation from Minkowski Space, JHEP 12 (2014) 151 [1410.0882].

[47] S. Nishi and T. Kobayashi, Generalized Galilean Genesis, JCAP 03 (2015) 057 [1501.02553].

[48] T. Kobayashi, M. Yamaguchi and J. Yokoyama, Galilean Creation of the Inflationary Universe, JCAP 07 (2015) 017 [1504.05710].
[49] Y. Cai and Y.-S. Piao, The slow expansion with nonminimal derivative coupling and its conformal dual, JHEP 03 (2016) 134 [1601.07031].

[50] S. Nishi and T. Kobayashi, Scale-invariant perturbations from null-energy-condition violation: $A$ new variant of Galilean genesis, Phys. Rev. D 95 (2017) 064001 [1611.01906].

[51] V. Volkova, S. Mironov and V. Rubakov, Cosmological Scenarios with Bounce and Genesis in Horndeski Theory and Beyond, J. Exp. Theor. Phys. 129 (2019) 553.

[52] A. Ilyas, M. Zhu, Y. Zheng and Y.-F. Cai, Emergent Universe and Genesis from the DHOST Cosmology, 2009.10351.

[53] V. Rubakov, The Null Energy Condition and its violation, Usp. Fiz. Nauk 184 (2014) 137 [1401.4024].

[54] E. Copeland, D. Mulryne, N. Nunes and M. Shaeri, Super-inflation in Loop Quantum Cosmology, Phys. Rev. D 77 (2008) 023510 [0708.1261].

[55] E. Copeland, D. Mulryne, N. Nunes and M. Shaeri, The gravitational wave background from super-inflation in Loop Quantum Cosmology, Phys. Rev. D 79 (2009) 023508 [0810.0104].

[56] Y. Mishima and T. Kobayashi, Revisiting slow-roll dynamics and the tensor tilt in general single-field inflation, Phys. Rev. D 101 (2020) 043536 [1911.02143].

[57] G. Capurri, N. Bartolo, D. Maino and S. Matarrese, Let Effective Field Theory of Inflation flow: stochastic generation of models with red/blue tensor tilt, 2006.10781.

[58] Y. Cai, Y.-T. Wang and Y.-S. Piao, Is there an effect of a nontrivial $c_{T}$ during inflation?, Phys. Rev. D 93 (2016) 063005 [1510.08716].

[59] Y. Cai, Y.-T. Wang and Y.-S. Piao, Propagating speed of primordial gravitational waves and inflation, Phys. Rev. D 94 (2016) 043002 [1602.05431].

[60] P. Creminelli, J. Gleyzes, J. Noreña and F. Vernizzi, Resilience of the standard predictions for primordial tensor modes, Phys. Rev. Lett. 113 (2014) 231301 [1407.8439].

[61] A. Gruzinov, Elastic inflation, Phys. Rev. D 70 (2004) 063518 [astro-ph/0404548].

[62] S. Endlich, A. Nicolis and J. Wang, Solid Inflation, JCAP 10 (2013) 011 [1210.0569].

[63] D. Cannone, G. Tasinato and D. Wands, Generalised tensor fluctuations and inflation, JCAP 01 (2015) 029 [1409.6568].

[64] N. Bartolo, D. Cannone, A. Ricciardone and G. Tasinato, Distinctive signatures of space-time diffeomorphism breaking in EFT of inflation, JCAP 03 (2016) 044 [1511.07414].

[65] A. Ricciardone and G. Tasinato, Primordial gravitational waves in supersolid inflation, Phys. Rev. D 96 (2017) 023508 [1611.04516].

[66] T. Fujita, S. Kuroyanagi, S. Mizuno and S. Mukohyama, Blue-tilted Primordial Gravitational Waves from Massive Gravity, Phys. Lett. B 789 (2019) 215 [1808.02381].

[67] A. Ashoorioon, K. Dimopoulos, M. Sheikh-Jabbari and G. Shiu, Non-Bunch-Davis initial state reconciles chaotic models with BICEP and Planck, Phys. Lett. B 737 (2014) 98 [1403.6099].

[68] C. Armendariz-Picon, T. Damour and V. F. Mukhanov, 
k-inflation, Phys. Lett. B 458 (1999) 209 [hep-th/9904075].

[69] M. Libanov, S. Mironov and V. Rubakov, Generalized Galileons: instabilities of bouncing and Genesis cosmologies and modified Genesis, JCAP 08 (2016) 037 [1605.05992].

[70] T. Kobayashi, Generic instabilities of nonsingular cosmologies in Horndeski theory: A no-go theorem, Phys. Rev. D 94 (2016) 043511 [1606.05831].

[71] P. Creminelli, D. Pirtskhalava, L. Santoni and E. Trincherini, Stability of Geodesically Complete Cosmologies, JCAP 11 (2016) 047 [1610.04207].

[72] Y. Cai, Y. Wan, H.-G. Li, T. Qiu and Y.-S. Piao, The Effective Field Theory of nonsingular cosmology, JHEP 01 (2017) 090 [1610.03400].

[73] Y. Cai, H.-G. Li, T. Qiu and Y.-S. Piao, The Effective Field Theory of nonsingular cosmology: II, Eur. Phys. J. C 77 (2017) 369 [1701.04330].

[74] K. Schmitz, New Sensitivity Curves for Gravitational-Wave Experiments, 2002.04615.

[75] BICEP2, KeCK ArRAY collaboration, P. Ade et al., BICEP2 / Keck Array x: Constraints on Primordial Gravitational Waves using Planck, WMAP, and New BICEP2/Keck Observations through the 2015 Season, Phys. Rev. Lett. 121 (2018) 221301 [1810.05216].

[76] B. Allen, The Stochastic gravity wave background: Sources and detection, in Les Houches School of Physics: Astrophysical Sources of Gravitational
Radiation, pp. 373-417, 4, 1996, gr-qc/9604033.

[77] M. Maggiore, Gravitational wave experiments and early universe cosmology, Phys. Rept. 331 (2000) 283 [gr-qc/9909001].

[78] T. L. Smith, E. Pierpaoli and M. Kamionkowski, A new cosmic microwave background constraint to primordial gravitational waves, Phys. Rev. Lett. 97 (2006) 021301 [astro-ph/0603144].

[79] L. A. Boyle and A. Buonanno, Relating gravitational wave constraints from primordial nucleosynthesis, pulsar timing, laser interferometers, and the $C M B$ :

Implications for the early Universe, Phys. Rev. D 78 (2008) 043531 [0708.2279].

[80] R. J. Cooke, M. Pettini and C. C. Steidel, One Percent Determination of the Primordial Deuterium Abundance, Astrophys. J. 855 (2018) 102 [1710.11129].

[81] T. Hsyu, R. J. Cooke, J. X. Prochaska and M. Bolte, The PHLEK Survey: A New Determination of the Primordial Helium Abundance, Astrophys. J. 896 (2020) 77 [2005.12290].

[82] Planck collaboration, N. Aghanim et al., Planck 2018 results. VI. Cosmological parameters, Astron. Astrophys. 641 (2020) A6 [1807.06209].

[83] ACT collaboration, S. Aiola et al., The Atacama Cosmology Telescope: DR4 Maps and Cosmological Parameters, 2007.07288. 